\title{
RESEARCH
}

Open Access

\section{RANKL blockade alleviates peri-implant bone loss and is enhanced by anti- inflammatory microRNA-146a through TLR2/4 signaling}

Keqing Pan ${ }^{1,2}$, Yang Hu${ }^{2}$, Yufeng Wang ${ }^{2,3}$, Hao $\mathrm{Li}^{2,4}$, Michele Patel ${ }^{2}$, Danyang Wang ${ }^{5}$, Zuomin Wang ${ }^{5}$ and Xiaozhe $\operatorname{Han}^{2^{*}}$

\begin{abstract}
Background: The present study was to determine the effect of local anti-RANKL antibody administration in the presence or absence of microRNA-146a on ligature-induced peri-implant bone resorption, and the potential role of TLR2/4 signaling in such effect.

Results: Titanium implants were placed in the left maxilla alveolar bone 6 weeks after extraction of first and second molars in C57/BL6 wild-type (WT) and TLR2 ${ }^{-1-} \mathrm{TLR}^{-/-}$(TLR2/4 KO) mice. Silk ligatures were tied around the implants 4 weeks after implantation. Anti-RANKL antibody $(500 \mu \mathrm{g} / \mathrm{mL}$ ) with or without microRNA 146a (miR-146a) (100 nM) was injected into palatal gingiva around implant on days 3, 6, and 9 during 2 weeks of ligation period. Bone resorption around the implants was assessed by $2 \mathrm{D}$ imaging using area measurement and 3D imaging using micro-computed tomography $(\mu \mathrm{CT})$. Real-time quantitative PCR (RT-qPCR) was used to determine the peri-implant gingival mRNA expression levels of pro-inflammatory cytokines (TNF-a) and osteoclastogenesis-related cytokines (RANKL). In both WT and TLR2/4 KO mice, the bone resorption around implants was significantly increased in the ligation only group when compared to the non-ligation group, but TLR2/4 KO mice showed significantly less bone loss compared to WT mice after ligation. As expected, gingival injection of anti-RANKL antibody significantly reduced bone loss compared with the ligation only group in both WT and TLR2/4 KO mice. Moreover, injection of miR-146a in addition to anti-RANKL antibody significantly enhanced the inhibition of bone loss in WT mice but not in TLR2/4 KO mice. Gingival mRNA expressions of RANKL were significantly reduced by anti-RANKL antibody treatment in both WT and TLR2/4 KO mice but were not affected by the additional miR-146a treatment. Gingival mRNA expression of TNF-a was significantly reduced by miR-146a treatment in WT mice but not in TLR2/4 KO mice. The number of gingival inflammatory cell infiltration and peri-implant TRAP-positive cell formation was significantly reduced by the additional miR-146a treatment in WT mice but not in TLR2/4 KO mice.
\end{abstract}

Conclusions: This study suggests that anti-inflammatory miR-146a enhance anti-RANKL-induced inhibition of periimplant bone resorption through the regulation of TLR2/4 signaling and inhibition of TNF-a expression.

Keywords: RANKL, Bone resorption, Peri-implantitis, MiR-146a, TLR2, TLR4

\footnotetext{
* Correspondence: Xhan@forsyth.org

${ }^{2}$ Department of Immunology and Infectious Diseases, The Forsyth Institute,

245 First Street, Cambridge, MA 02142, USA

Full list of author information is available at the end of the article
}

\section{Springer Open}

(c) The Author(s). 2020 Open Access This article is licensed under a Creative Commons Attribution 4.0 International License, which permits use, sharing, adaptation, distribution and reproduction in any medium or format, as long as you give appropriate credit to the original author(s) and the source, provide a link to the Creative Commons licence, and indicate if changes were made. The images or other third party material in this article are included in the article's Creative Commons licence, unless indicated otherwise in a credit line to the material. If material is not included in the article's Creative Commons licence and your intended use is not permitted by statutory regulation or exceeds the permitted use, you will need to obtain permission directly from the copyright holder. To view a copy of this licence, visit http://creativecommons.org/licenses/by/4.0/. 


\section{Background}

Dental implant has become a preferable choice to restore the missing tooth in the past few decades for functional and esthetic purposes [1]. However, peri-implantitis has become prevalent accompanying the exponential growth of dental implant procedures [2, 3]. Peri-implantitis is indicated by infection of implant surrounding soft tissues and bone loss, resulting in implant failure eventually [4-6]. The host immune and inflammatory responses caused by plaques on implant surface are crucial in the pathogenesis of peri-implantitis $[2,7,8]$. However, current treatment available for peri-implantitis is not satisfactory due to the lack of understanding of the mechanism of peri-implantitis pathogenesis.

Receptor activator of nuclear factor-kappa B (RANK) and its ligand RANKL and the decoy receptor osteoprotegerin (OPG) are central regulators of osteoclast development and essential for osteoimmunology [9-12]. Recent study showed that RANKL/OPG ratio was significantly increased in the gingival tissues surrounding mini-implants in the rat model with Porphyromonas gingivalis LPS inductions [13]. Moreover, anti-RANKL antibody was approved for the treatment of osteoporosis, and it showed inhibition of bone loss in rodent experimental periodontitis models [14-17]. Our previous study showed that administration of anti-RANKL antibody directly to the gingival of rat experimental periodontitis model can significantly reduce gingival sRANKL expression and of bone resorption [18]. However, the effects of anti-RANKL antibody on peri-implantitis have not been investigated.

MicroRNAs (miRs) are small non-coding RNA molecules found in plants, animals, and some viruses, functioning in RNA silencing and post-transcriptional regulation of gene expression [19-21]. Recent studies showed that miRs are important regulators in periodontitis [21-23]. Our previous studies demonstrated that miR-146a regulated the cytokine secretion in human gingival fibroblasts and periodontal ligament cells and inhibits inflammatory cytokine production in B cells through directly targeting IRAK1, suggesting a regulatory role of miR-146a in immune-mediated periodontal inflammation [24]. However, the role of miR-146a in peri-implantitis remains unknown.

Toll-like receptors (TLR) are a family of wellcharacterized pattern recognition receptors (PRRs) and play an important role in the induction of pro-inflammatory cytokines by recognizing the signature molecules of the host innate immunity [25-27]. Our previous studies showed that TLR2 are associated with implant bone loss in a mouse model of peri-implantitis [5] and TLR4 is essential for periodontal bone loss $[28,29]$. In our previous study, we examined the changes of inflammatory cytokines and bone metabolism cytokines in either TLR2 only $\mathrm{KO}$ mice or TLR4 only KO mice $[5,29]$. However, since anti-RANKL antibody and miR-146a may interact with both TLR2 and TLR4 pathways, TLR2 and TLR4 double knockout (TLR2/ $4 \mathrm{KO}$ ) mice were specially employed in the present study to determine whether the effects of local anti-RANKL antibody administration in the presence or absence of miR146a on ligature-induced peri-implant bone loss are dependent on both TLR2 and TLR4.

While our previous studies have substantiated that RANKL blockade inhibited immune-mediated RANKLdependent bone loss, others have indicated that proinflammatory cytokines, such as SOFAT and TNF-alpha, could induce osteoclastogenesis in a RANKL-independent manner [30-32] through TLR signaling pathway [33]. MiR-146 has been implicated in the involvement of the innate immune responses through negative feedback regulation of TLR signaling [34]. In particular, recent studies have concluded that miR-146a has a diverse and critical role in limiting an excessive acute inflammatory reaction [35]. The purpose of the current study is to investigate the potential synergistic effect of RANKL blockage and antiinflammatory miR-146a in the control of peri-implant bone loss. Our hypothesis is that anti-inflammatory microRNA-146a synergistically enhance anti-RANKL antibody-induced inhibition of peri-implant bone loss through TLR2/4 signaling.

\section{Methods}

Mice

Wild-type (WT) C57BL/6 and TLR2 KO and TLR4 KO mice in C57/BL6 background were purchased from the Jackson Laboratory (Bar Harbor, ME). TLR2 and TLR4 double $\mathrm{KO}$ mice (TLR2/4 KO) were crossbreed from TLR2 KO and TLR4 KO mice and confirmed by genotyping. All the animal-associated protocols were reviewed and approved (\#17-022) by the Institutional Animal Care and Use Committee of the Forsyth Institute. All the mice used in the study were maintained in specific pathogen-free units. Mice were fed a soft diet ad libitum for the duration of the experiment. Forty-eight WT mice and forty-eight TLR2/4 KO mice were used in this study, and the mice were randomly divided into 4 groups in WT and TLR2/4 KO mice as follows: (1) implant only $(n=12)$, (2) implant + ligation $(n=12)$, (3) implant + ligation+ anti-RANKL antibody $(n=12)$, and (4) implant + ligation + anti-RANKL antibody + miR$146 a(n=12)$.

\section{Tooth extraction, implant placement, and ligature- induced experimental peri-implantitis and local administration of anti-RANKL-antibody and miR-146a}

The procedures of tooth extraction and implant placement were as previously described [5]. Briefly, all the mice had their left maxillary first and second molars extracted at 4 weeks old with 6 weeks of healing time after 
the tooth extraction. Drinking water with antibiotics (sulfamethoxazole and trimethoprim, $850 \mu \mathrm{g} / 170 \mu \mathrm{g}$ per $\mathrm{mL}$ ) was used for 2 weeks to decrease the possibility of infection after tooth extraction. Then, the maxillary alveolar bone was drilled with the 0.3-mm-diameter carbide micro-hand drill, and a smooth-surface, screwshaped titanium implant $(1 \mathrm{~mm}$ in length and $0.5 \mathrm{~mm}$ in diameter, D. P. Machining) was screwed clockwise into the bone through the drilled site until torque could be achieved. Implants were allowed to heal for 4 weeks, and the antibiotic water was given during the first week after implantation as described above. Four weeks after implant placement, experimental peri-implantitis was initiated with a 7-0 silk ligature tied around the implants. The ligation day was recorded as day 0 , and the ligature remained in place for 2 weeks. For group 3 and group 4, each mouse received palatal gingival injections of $2 \mu \mathrm{L}$ of anti-RANKL antibody $(500 \mu \mathrm{g} / \mathrm{mL}$, Peprotech, Rocky Hill, NJ) or miR-146a (100 nM, GeneCopoeia, Rockville, $\mathrm{MD}$ ) on the mesial and distal gingival papillae of implant by a 31-gauge double-beveled MicroFine needle (Becton, Dickinson) as these doses were established from our previous publications [24, 28]. The injections for animals were administered three times on days 3,6 , and 9 , and all the mice were euthanized by $\mathrm{CO}_{2}$ inhalation on day 14. All the procedures, including tooth extraction, implant placement, ligature placement, and injection, were performed using an optical microscope (S6D Stereozoom, Leica).

\section{Tissue collection and sample preparation}

The ligations were maintained for 2 weeks, and after which the mice were euthanized by $\mathrm{CO}_{2}$ inhalation and the maxilla were harvested. The gingival tissues of half group of mice were isolated and collected for mRNA expression study. The skulls left were defleshed by beetles for 1 week. Briefly, in beetle's chamber, freshly dissected skull was put in a paper cup with $0.5 \mathrm{~cm}$ diameter holes at the bottom, so beetles can move in with relatively controlled numbers. After that, the skulls were bleached by $\mathrm{H}_{2} \mathrm{O}_{2}$ (3\%) for $4 \mathrm{~h}$. Bone resorption was measured by microscope imaging analysis and $\mu \mathrm{CT}$ scan analysis. The skulls of the other half of the group were fixed in formalin overnight at $4{ }^{\circ} \mathrm{C}$ followed by EDTA decalcification for 3 weeks with agitation. After complete demineralization, implants were removed manually by rotating counterclockwise. All the decalcification samples were embedded into paraffin and cut in $5 \mu \mathrm{m}$ sections along the mesial-distal plane and then subjected to H\&E staining and TRAP staining.

\section{Imaging analysis of bone resorption area}

The two dimension (2D) bone resorption measurements were assessed under a microscope (Nikon SMZ745T,
Nikon Instruments Inc.) and analyzed by software ImageJ (NIH) on buccal and palatal surfaces for each segment, and a standard calibrator was used for calibration at the same magnification as previously described [36]. The bone resorption area was enclosed coronally by the CEJ of the molars, laterally by the exposed distal root of the first molar and the exposed mesial root of the third molar, and apically by the alveolar crest. The results are presented in square millimeters.

\section{Micro-computed tomography analysis}

Mice maxillae were scanned with a high-resolution scanner (mCT-40, Scanco Medical). Samples were exposed to polychromatic X-rays on a rotating stage at a steep angle of $0.18^{\circ}$ over $360^{\circ}$. Measurements were taken at an operating voltage of $70 \mathrm{kVp}$ and $114 \mathrm{~mA}$ current and 6 $\mathrm{mm}$ isotropic voxel resolution, with an exposure time of $200 \mathrm{~ms}$ and five frames averaged per view. Quantitative three dimension (3D) measurements of the bone resorption were performed using Seg3D software as previously described [5].

\section{Real-time quantitative PCR}

Palatal gingival tissues were isolated from around ligatured implants and were homogenized in lysis buffer using a tissue homogenizer. Total RNA was extracted using PureLink $^{\bullet}$ RNA Mini Kit (Ambion). cDNA was synthesized using the SuperScript III Reversed Transcriptase kit (Invitrogen) according to the manufacturer's protocol. The mRNA expression of TNF- $\alpha$ and RANKL in gingival was determined by real-time quantitative PCR (RT-qPCR) using LightCycler ${ }^{\circ}$ SYBR Green I master and LightCycler ${ }^{\circ} 480$ Instrument system (Roche). GAPDH gene was used as an internal control. The sequences of primers are listed as follows: TNF- $\alpha$ forward 5'-CACAGAAAGCATGATCCG CGACGT-3'; TNF- $\alpha$ reverse 5'-CGGCAGAGAGGAGG TTGACTTTCT-3'; RANKL forward 5'-GGGTGTGTAC AAGACCC-3'; RANKL reverse 5'-CATGTGCCACTGAG AACCTTGAA-3" GAPDH forward 5'-CCCCAGCAAG GACACTGAGCAA-3'; GAPDH reverse 5'-GTGGGT GCAGCGAACTTTATTGATG-3' .

\section{Hematoxylin and eosin staining and tartrate-resistant acid phosphatase staining}

All the maxilla collected were fixed in $4 \%$ formaldehyde overnight and then went through decalcification in $10 \%$ EDTA for 3 weeks at $4{ }^{\circ} \mathrm{C}$ with shaking. Five-micrometerthick sections were produced in the mesial-distal plane for hematoxylin and eosin (H\&E) and tartrate-resistant acid phosphatase (TRAP) staining. An acid phosphatase kit (catalog number 387A, Sigma) was used for TRAP staining. After $30 \mathrm{~min}$ staining and $1 \mathrm{~min}$ counterstain with hematoxylin, TRAP-positive cells with three or more nuclei were considered to be osteoclasts. A region of interest 
(ROI) was defined in peri-implantitis samples as a $1.5 \times 1$ $\mathrm{mm}$ rectangular area aligned with the central long axis of the implant and covered the whole length of the implant. TRAP-positive cell numbers within the ROI were quantified manually by ImageJ. For H\&E staining, images were analyzed by ImageJ after being captured by a digital camera. The numbers of inflammatory cells from the implant supportive tissue on each slide were counted at a magnification of $\times 40$, and the average numbers were calculated.

\section{Statistical analysis}

Results were presented as mean $\pm \mathrm{SD}$. Unpaired Student's $t$ test was used to analyze differences between any two groups of data sets. Results with $p<0.05$ are considered statistically significant.

\section{Results}

\section{Ligature-induced peri-implant inflammation and bone} loss in mice

In our ligature-induced experimental peri-implantitis mouse model, teeth extraction, implant placement, ligation placement, and gingival injection will be performed in a 12-week process (Fig. 1). 86.67\% (52 out of 60) of implants in TLR2/4 KO mice achieved osteointegration (no mobility when touched by needles, no obvious bleeding upon probing) after being placed for 4 weeks, which has no significant difference with WT mice success rate $81.67 \%$ (Table 1 ). The gingival tissue surrounding the implant appeared healthy (pink in color, no obvious swelling, no bleeding upon probing) 28 days after implant placement in WT and TLR2/4 KO mice, but gingival tissue turned red in color and displayed apparent swelling 3 days after the ligature was placed. Furthermore, the ligation group showed a significantly higher bone resorption compared with the non-ligation group in WT mice, for both $2 \mathrm{D}$ imaging analysis $(p=$ 0.0021 , Fig. 2a, b) and $\mu \mathrm{CT}$ analysis $(p=0.0012$, Fig. $2 \mathrm{~d})$, and in TLR2/4 KO mice for both 2D imaging analysis ( $p=$ 0.0039, Fig. 2a, c) and $\mu \mathrm{CT}$ analysis $(p=0.0023$, Fig. 2e). Taken together, these results indicate that ligature successfully induced inflammation and bone resorption in this mouse model of peri-implantitis.

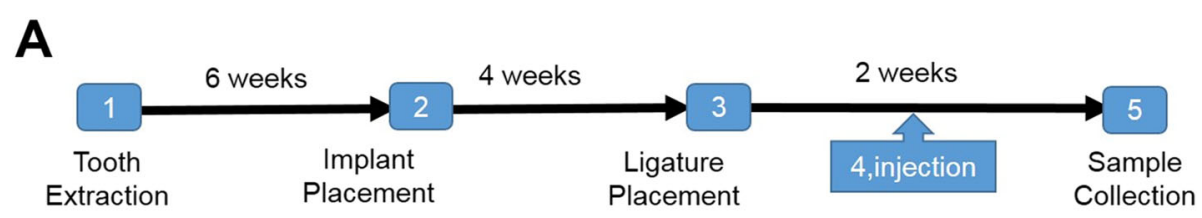

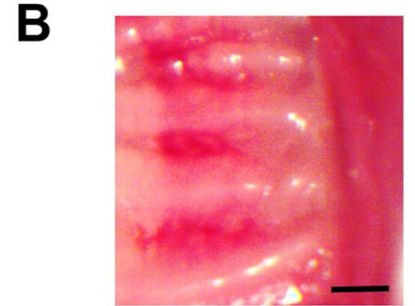

6 weeks after tooth extraction

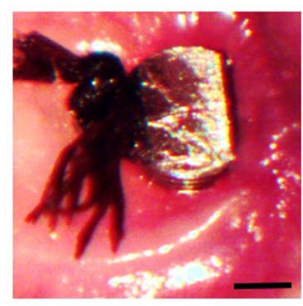

Ligation day 0

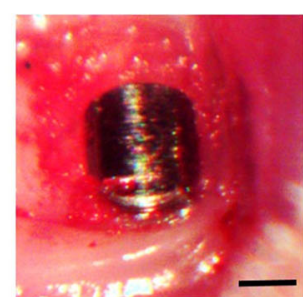

Implantation day 0

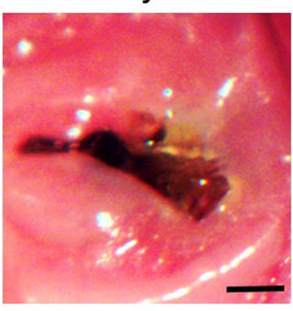

Injection at postligation day $3,6,9$

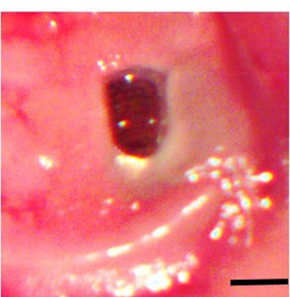

4 weeks post-Implantation

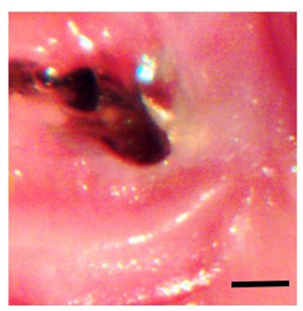

2 weeks post-ligation

Fig. 1 Mouse model of ligature-induced experimental peri-implantitis. (a) Tooth extraction: left maxillary first and second molars extracted at 4 weeks old and the tooth extraction socket healed well with smooth gingiva surface after 6 weeks post-extraction. Implant placement: implant was put in alveolar bone without flap elevation. Ligature placement: at 4 weeks post-implant, 7-0 ligatures were applied under the fixture head. Gingival injection: injections for animals were administered three times on days 3, 6, and 9 during 14 days ligation period. Sample collection: 14 days post-ligation, the gingival tissues and the skulls were collected. (b) Images depicting processing steps of the experimental design (scale bar, $500 \mu \mathrm{m}$ ) 
Table 1 Success rate (SR) of osseointegrated implants 4 weeks after implant placement

\begin{tabular}{lllllll}
\hline & Total implants & Lost & Loose & Osseointegrated & Success rate $(\%)$ & SR $P$ value \\
\hline Wild type group & 60 & 6 & 5 & 49 & 81.67 & 0.595 \\
TLR2/4 KO group & 60 & 4 & 4 & 52 & 86.67
\end{tabular}

\section{Anti-RANKL antibody and anti-RANKL+miR-146a treatments showed different effects on peri-implantitis bone loss in WT and TLR2/4 KO mice}

Anti-RANKL antibody alone significantly reduced bone loss compared with the ligation only group in both WT and TLR2/4 KO mice; however, injection of miR-146a in addition to anti-RANKL antibody significantly enhanced the inhibition of bone loss in WT mice but not in TLR2/4 KO mice (Fig. 2b-e). Significantly higher number of osteoclasts were observed in the ligation group vs. non-ligation group in WT mice and TLR2/4 KO mice in TRAP staining (Fig. 3a-c), which is consistent with the results of $2 \mathrm{D}$ and $3 \mathrm{D}$ bone loss analysis (Fig. 2b-e). Moreover, the antiRANKL+miR-146a treatment groups showed significantly lower number of osteoclasts compared with the anti-RANKL treatment group in WT mice but not in TLR2/4 KO mice (Fig. 3b, c), which is also consistent with the results of bone loss analysis (Fig. 2b-e). Taken together, miR-146a in addition to anti-RANKL antibody can further reduce bone loss in WT mice but is ineffective when TLR 2 and TLR4 are deficient, suggesting miR-146a anti-bone loss effects in periimplantitis are TLR2/4 dependent.

\section{Anti-RANKL antibody and anti-RANKL+miR-146a treatments had different effects on peri-implantitis inflammation in WT and TLR2/4 KO mice}

In both WT mice and TLR $2 / 4 \mathrm{KO}$ mice, a significantly higher number of inflammatory cells were found infiltrating around the peri-implant tissues in the ligation group compared with the non-ligation group (Fig. $4 \mathrm{a}-\mathrm{c}$ ). However, the number of inflammatory cells in tissues of the ligation group was not significantly changed when treated with anti-RANKL antibody alone in both WT and TLR2/4 KO mice compared with the ligation group (Fig. 4b, c). MiR146a treatment additional to anti-RANKL antibody significantly decreased the number of inflammatory cells in WT mice but not in TLR2/4 KO mice when compared with the anti-RANKL antibody alone group. Taken together, anti-RANKL antibody alone did not affect inflammatory cell infiltration in peri-implantitis, and miR-146a showed anti-inflammatory effects only on WT mice but not on TLR2/4-deficient mice.
Pro-inflammatory and bone metabolism factors showed different pattern of change when treated with anti-RANKL antibody and anti-RANKL+miR-146a in WT and TLR2/4 KO mice

Gingival TNF- $\alpha$ mRNA showed a significant upregulation in the ligation group compared with the non-ligation group in both WT and TLR2/4 KO mice (Fig. 5a, b). Moreover, TNF- $\alpha$ showed no significant decrease when treated with anti-RANKL antibody alone in both WT and TLR2/4 KO mice compared with the ligation group. However, additional miR-146a treatment significantly decreased TNF- $\alpha$ expression in WT mice but not in TLR2/4 $\mathrm{KO}$ mice (Fig. 5a, b), suggesting the consistent results of anti-inflammation effects characterized by quantification of infiltrating inflammation cells (Fig. $4 \mathrm{a}-\mathrm{c}$ ). Meanwhile, gingival RANKL mRNA expression was significantly decreased with anti-RANKL antibody alone in both WT and TLR2/4 KO mice, and additional miR-146a treatment did not show significant difference compared with the antiRANKL antibody treatment group (Fig. 5c, d). Taken together, anti-RANKL antibody showed the inhibition effects on RANKL expression in both WT and TLR2/4 KOTLR2/4 KO mice, and miR-146a showed antiinflammation effect through downregulation of TNF- $\alpha$ mRNA only in WT mice.

\section{Discussion}

Our present study showed that anti-RANKL antibody can significantly inhibit the bone loss in peri-implantitis and additional miR-146a treatment will enhance this inhibition through its anti-inflammation effects via TLR2/4 signaling. This is the first report in a murine model of periimplantitis to demonstrate that anti-RANKL antibody and miR-146a together can significantly reduce bone resorption and inflammation in peri-implantitis, suggesting a potential therapeutic strategy for peri-implantitis patients. Moreover, the data showed that anti-bone loss effects of anti-RANKL antibody are independent of TLR2 and TLR4 and anti-RANKL antibody alone did not affect periimplant inflammatory infiltration, suggesting that RANKL modulation of bone loss is downstream of peri-implant inflammation and more directly towards bone resorption. However, anti-RANKL antibody + miR-146a treatment showed significantly stronger inhibition of bone loss than anti-RANKL antibody alone treatment, indicating that the suppression of inflammation can be used to reduce peri- 


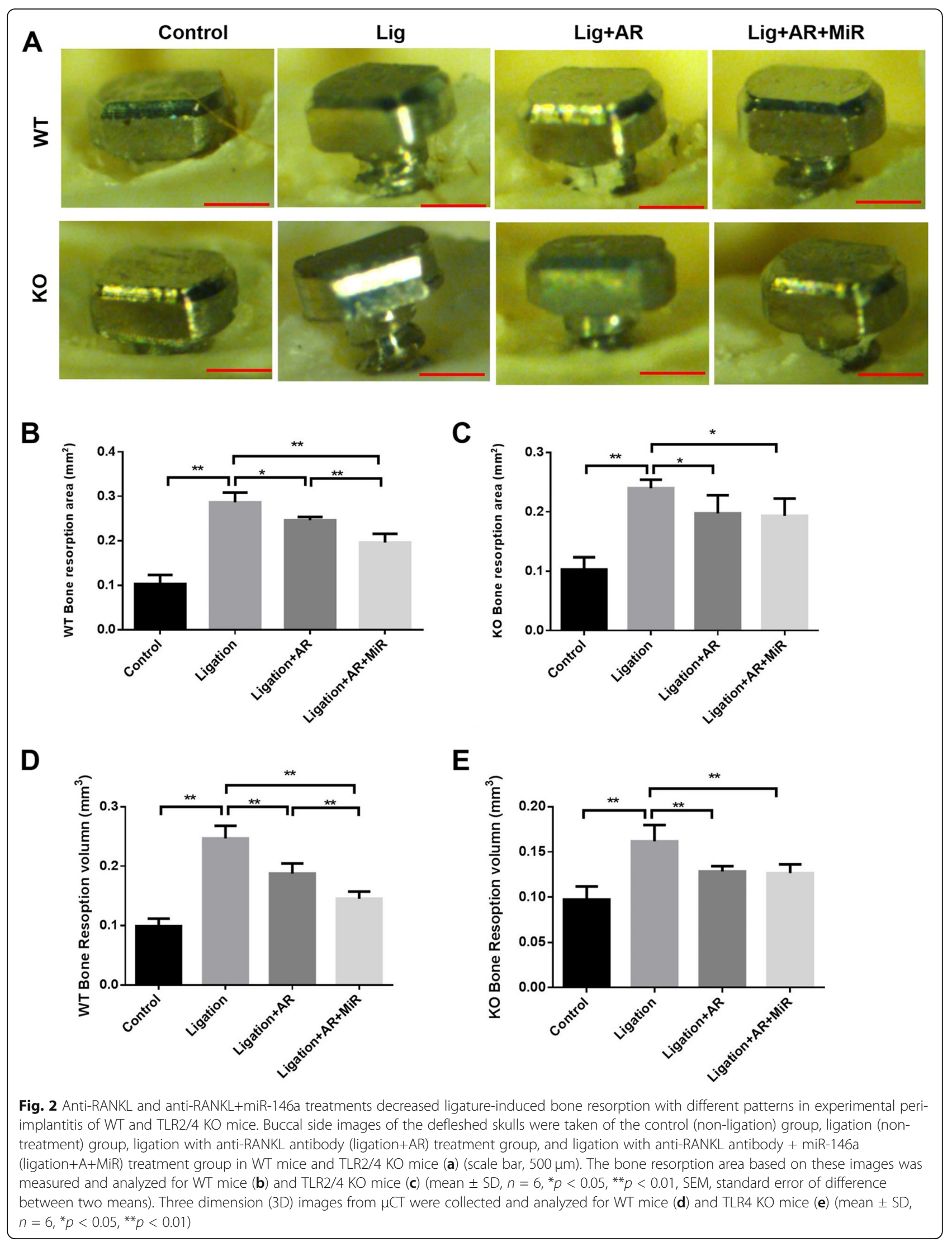




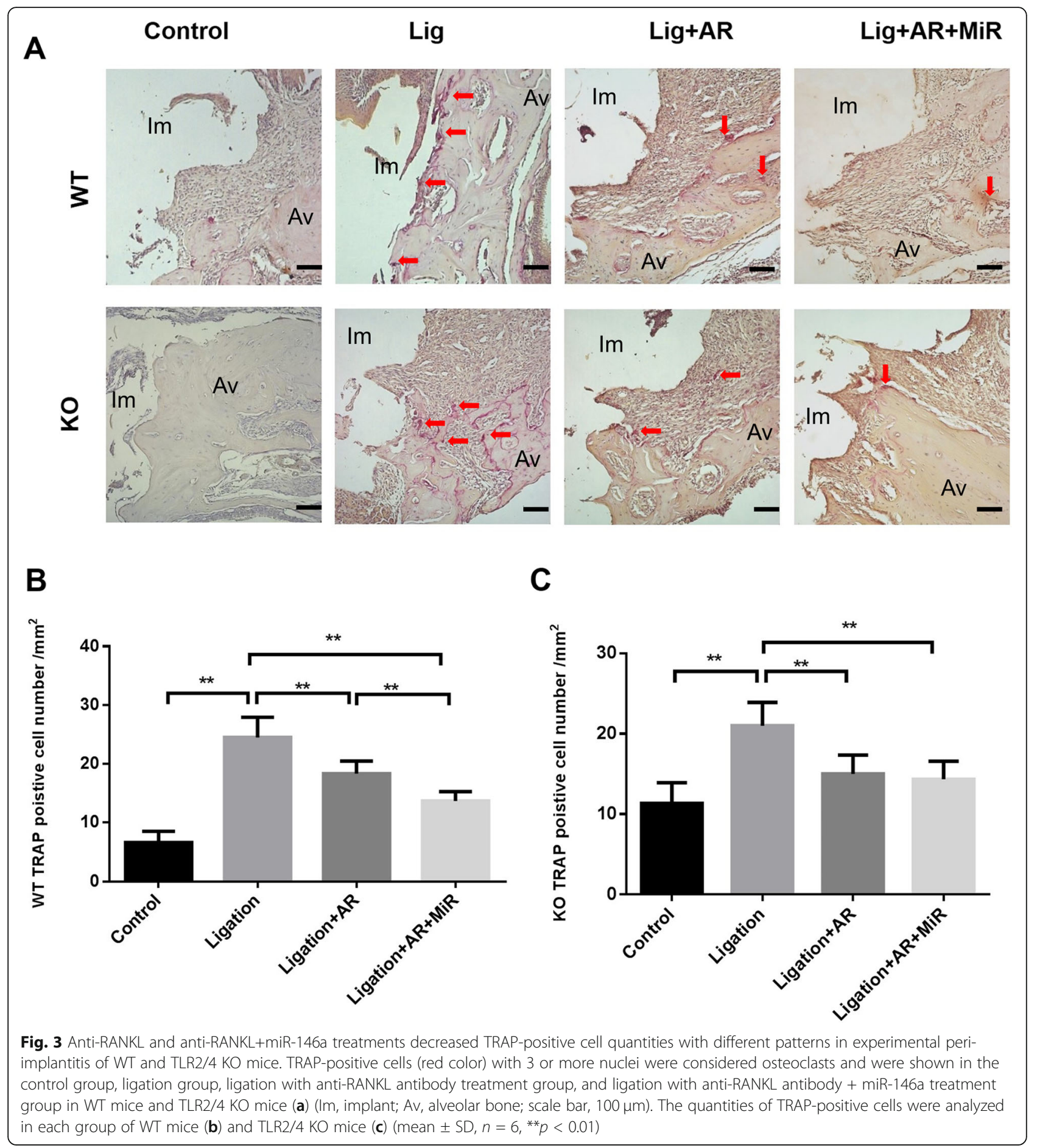

implantitis bone loss by removing additional RANKLindependent etiology and pathogenesis of bone loss. These data (Fig. 1-5, Supplemental Table S1) showed that antiinflammatory miR-146a enhance anti-RANKL-induced inhibition of peri-implant bone resorption through the regulation of TLR2/4 signaling and inhibition of TNF- $\alpha$ expression. Thus, the ideal osteoimmunological treatment for peri-implantitis should include both direct antiosteoclastogenesis and anti-inflammation components.

As innate immune recognition receptors, TLR family plays a central role in innate immunity, inflammation, cell survival, and proliferation [5, 26, 37, 38]. TLR2 and TLR4 


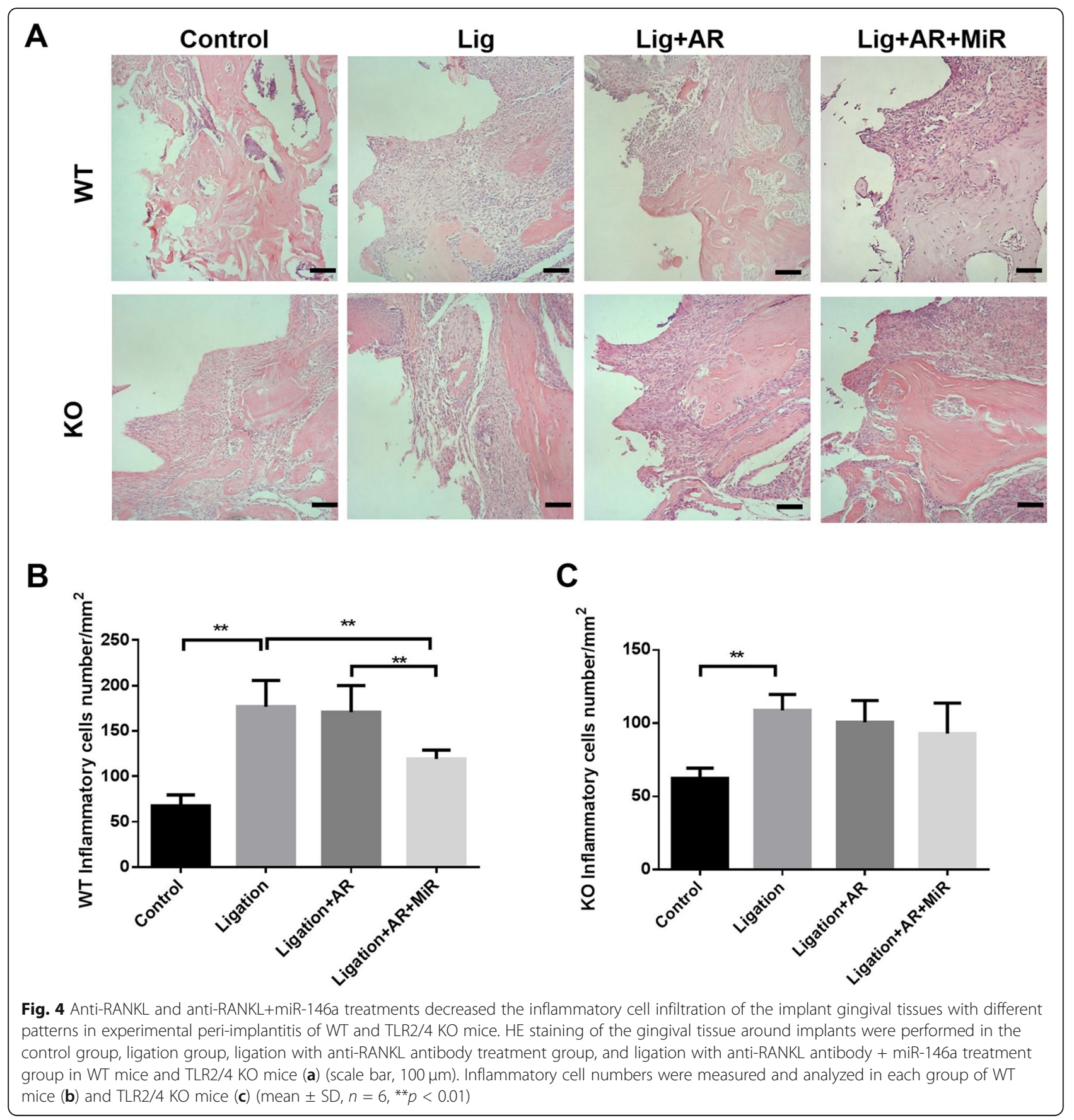

are essential signaling proteins in progression of inflammation and related bone metabolism in periodontitis [28, 29, 39, 40]. However, little is known about the functions of TLR2 and TLR4 signaling in peri-implantitis. In the present study, we investigated the changes in bone resorption, gingival TNF- $\alpha$ (pro-inflammatory marker) mRNA levels, and gingival soluble RANKL protein levels in ligation-induced experimental periimplantitis in WT and TLR2/4 KO mice with or without anti-RANKL antibody alone treatment and anti-RANKL antibody + miR-146a treatment. The results showed that anti-bone loss effects of anti-RANKL antibody are TLR2/4 independent but anti-inflammation and related anti-bone loss effects of miR-146a are TLR2/4 dependent, suggesting that TLR2/4 signaling is crucial for RANKL-independent, inflammation-induced bone loss in peri-implantitis.

According to the previous studies, miR-146a was regulated by NF- $\mathrm{kB}$ and blockade of miR-146a could decrease TLR4 and NF-kB in human cells [41, 42], suggesting that miR-146a is involved in TLR/NF-kB signaling pathway. 


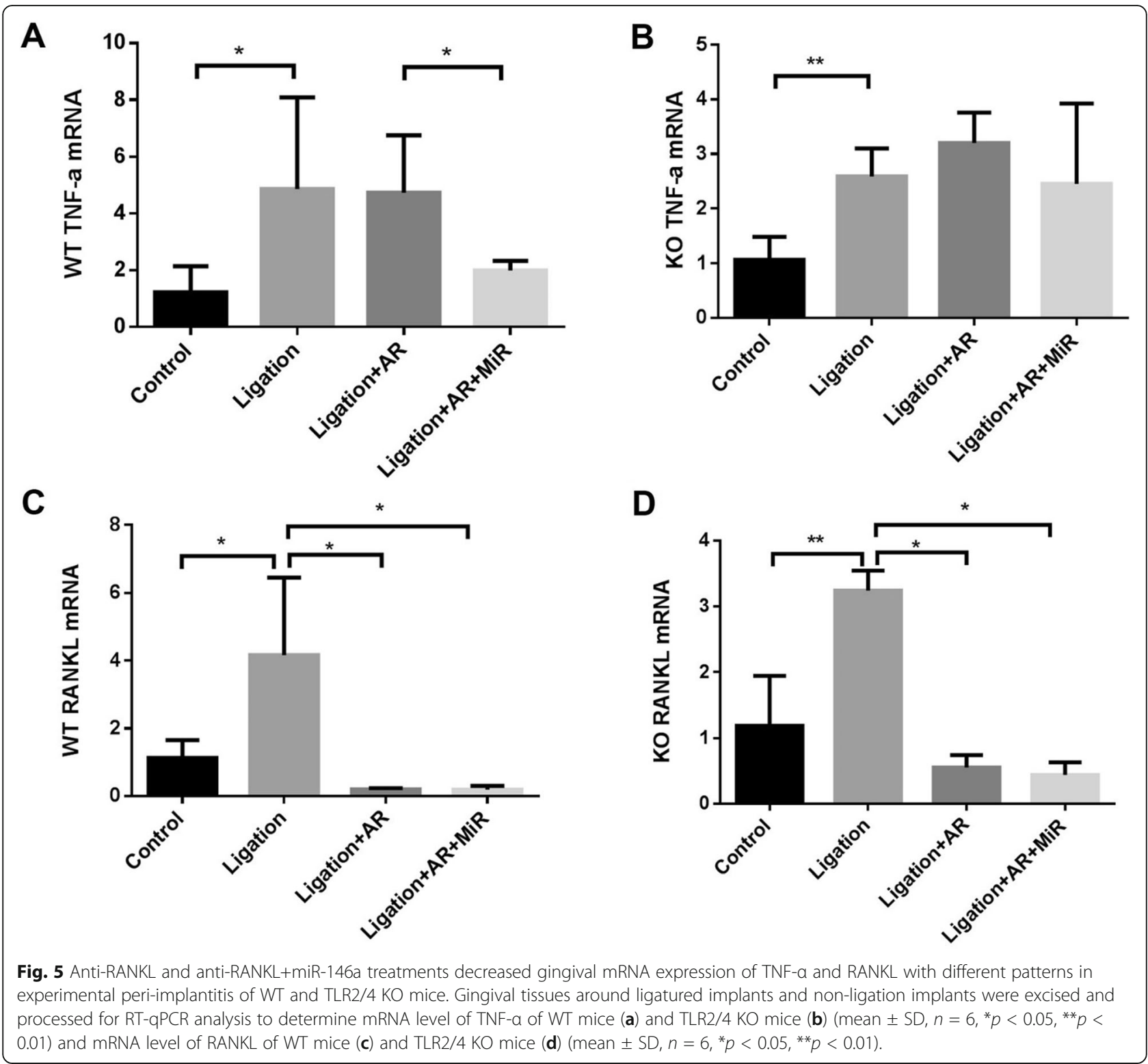

Our recent study showed that miR-146a inhibited inflammatory cytokine secretion in B cells after challenged with $P$. gingivalis LPS and decreased bone resorption in experimental periodontits animal models [24]. Moreover, it was found that miR-146a negatively regulated TLR2-induced inflammatory response in keratinocytes [43] and expression of TLR2 was repressed by miR-146a in HEK293T cells [44]. Thus, the cross talk between miR-146a and TLR2/4 may be essential for anti-inflammation effects of miR-146a by inhibiting NF- $\mathrm{KB}$ signaling. In the present study, the data showed that miR-146a have no effects on inflammatory cell infiltration or TNF- $\alpha$ expression in the absence of TLR2 and TLR4 in experimental peri-implantitis, suggesting that miR-146a anti-inflammation effects are TLR2/4 dependent in peri-implantitis. However, on the other hand, how induction of TLR2/4 in oral disease affects the expression and function of miR-146a may need further investigation.

\section{Conclusions}

In summary, the present study suggests that antiinflammatory miR-146a enhance anti-RANKL-induced inhibition of peri-implant bone resorption through the regulation of TLR2/4 signaling and inhibition of TNF- $\alpha$ expression. Combination of regimens antagonizing both osteoclastogenesis and inflammation may become a more effective strategy to ameliorate peri-implantitis bone loss. 


\section{Supplementary information}

Supplementary information accompanies this paper at https://doi.org/10. 1186/s40729-020-00210-0.

Additional file 1: Supplemental Table S1. The numerical data of all graphs.

\section{Abbreviations}

RANKL: Receptor activator of nuclear factor-kappa B ligand; TLR: Toll-like receptor; WT: Wild type; miR-146a: MicroRNA 146a; MCT: Micro-computed tomography; RT-qPCR: Real-time quantitative PCR; TNF-a: Tumor necrosis factor alpha; OPG: Osteoprotegerin; LPS: Lipopolysaccharides; H\&E: Hematoxylin and eosin; TRAP: Tartrate-resistant acid phosphatase

\section{Acknowledgements}

Not applicable

\section{Authors' contributions}

KP contributed to the conception and design; contributed to the acquisition analysis, and interpretation of data; and drafted the manuscript. YH contributed to the conception and design, contributed to the acquisition, analysis, and interpretation of data; drafted the manuscript; and critically revised the manuscript for important intellectual content. YW contributed to the conception and design and to the acquisition, analysis, and interpretation of data. HL contributed to the acquisition, analysis, and interpretation of data. PM contributed to the acquisition, analysis, and interpretation of data. DW critically revised the manuscript for important intellectual content. ZW critically revised the manuscript for important intellectual content. XH contributed to the conception and design; contributed to the acquisition, analysis, and interpretation of data; critically revised the manuscript for important intellectual content, and agree to be accountable for all aspects of the work in ensuring that questions relating to the accuracy or integrity of any part of the work are appropriately investigated and resolved. The authors read and approved the final manuscript.

\section{Funding}

This study was supported by NIH NIDCR R01DE025255 and the Forsyth Institute FPILOT36 to X Han and the Forsyth Institute FPILOT52 to Y Hu.

\section{Availability of data and materials}

Presented in the main paper

\section{Ethics approval and consent to participate}

Not applicable

\section{Consent for publication}

Not applicable

\section{Competing interests}

Keqing Pan, Yang Hu, Yufeng Wang, Hao Li, Michele Patel, Danyang Wang, Zuomin Wang, and Xiaozhe Han certify that they do not have any commercial or associate interest that represents a conflict of interest in connection with the manuscript. The submitted work was not carried out in the presence of any personal, professional, or financial relationships that could potentially be construed as a conflict of interest.

\section{Author details}

${ }^{1}$ Department of Stomatology, The Affiliated Hospital of Qingdao University, College of Stomatology, Qingdao University, Qingdao 266003, Shandong, China. ${ }^{2}$ Department of Immunology and Infectious Diseases, The Forsyth Institute, 245 First Street, Cambridge, MA 02142, USA. ${ }^{3}$ Department of Oral Medicine, Ninth People's Hospital, National Clinical Research Center of Stomatology, , Shanghai Jiao Tong University School of Medicine; Shanghai Key Laboratory of Stomatology \& Shanghai Research Institute of Stomatology, Shanghai 200011, China. ${ }^{4}$ Department of Prosthodontics, The Affiliated Hospital of Stomatology, Guangxi Medical University, Nanning 530021, China. ${ }^{5}$ Department of Stomatology, Beijing ChaoYang Hospital affiliated with Capital Medical University, Beijing, China.
Received: 24 September 2019 Accepted: 12 March 2020

Published online: 15 April 2020

\section{References}

1. Bertin TJC, Thivichon-Prince B, LeBlanc ARH, Caldwell MW, Viriot L. Current perspectives on tooth implantation, attachment, and replacement in amniota. Front Physiol. 2018;9:1630

2. Schminke B, Vom Orde F, Gruber R, Schliephake H, Burgers R, Miosge N. The pathology of bone tissue during peri-implantitis. J Dent Res. 2015;94(2):354-61.

3. Mombelli A, Muller N, Cionca N. The epidemiology of peri-implantitis. Clin Oral Implants Res. 2012;23(Suppl 6):67-76.

4. Colombo APV, Tanner ACR. The role of bacterial biofilms in dental caries and periodontal and peri-implant diseases: a historical perspective. J Dent Res. 2019;98(4):373-85.

5. Yu X, Hu Y, Freire M, Yu P, Kawai T, Han X. Role of toll-like receptor 2 in inflammation and alveolar bone loss in experimental peri-implantitis versus periodontitis. J Periodontal Res. 2017.

6. Tzach-Nahman R, Mizraji G, Shapira L, Nussbaum G, Wilensky A. Oral infection with Porphyromonas gingivalis induces peri-implantitis in a murine model: evaluation of bone loss and the local inflammatory response. J Clin Periodontol. 2017;44(7):739-48.

7. Nguyen Vo TN, Hao J, Chou J, et al. Ligature induced peri-implantitis: tissue destruction and inflammatory progression in a murine model. Clin Oral Implants Res. 2017;28(2):129-36.

8. Carcuac O, Berglundh T. Composition of human peri-implantitis and periodontitis lesions. J Dent Res. 2014:93(11):1083-8.

9. Weitzmann MN. The role of inflammatory cytokines, the RANKL/OPG axis, and the immunoskeletal interface in physiological bone turnover and osteoporosis. Scientifica (Cairo). 2013;2013:125705.

10. Sano T, Akeda K, Yamada J, Takegami N, Sudo T, Sudo A. Expression of the RANK/RANKL/OPG system in the human intervertebral disc: implication for the pathogenesis of intervertebral disc degeneration. BMC Musculoskelet Disord. 2019:20(1):225

11. Chen Y, Yang K, Zhou Z, Wang L, Du Y, Wang X. Mechanical stress modulates the RANKL/OPG system of periodontal ligament stem cells via alpha7 nAChR in human deciduous teeth: an in vitro study. Stem Cells Int 2019:2019:5326341.

12. Amin N, Boccardi V, Taghizadeh M, Jafarnejad S. Probiotics and bone disorders: the role of RANKL/RANK/OPG pathway. Aging Clin Exp Res. 2019.

13. Shuto T, Wachi T, Shinohara Y, Nikawa H, Makihira S. Increase in receptor activator of nuclear factor kappaB ligand/osteoprotegerin ratio in periimplant gingiva exposed to Porphyromonas gingivalis lipopolysaccharide. J Dent Sci. 2016;11(1):8-16.

14. O'Connell MB. Prescription drug therapies for prevention and treatment of postmenopausal osteoporosis. J Manag Care Pharm. 2006;12(6 Suppl A):S1019; quiz S26-18.

15. O'Connor KM. Evaluation and treatment of osteoporosis. Med Clin North Am. 2016:100(4):807-26.

16. O'Halloran M, Boyd NM, Smith A. Denosumab and osteonecrosis of the jaws - the pharmacology, pathogenesis and a report of two cases. Aust Dent J. 2014;59(4):516-9.

17. Han $X$, Lin $X, Y u X$, et al. Porphyromonas gingivalis infection-associated periodontal bone resorption is dependent on receptor activator of NFkappaB ligand. Infect Immun. 2013:81(5):1502-9.

18. Lin X, Han X, Kawai T, Taubman MA. Antibody to receptor activator of NFkappaB ligand ameliorates T cell-mediated periodontal bone resorption. Infect Immun. 2011;79(2):911-7.

19. Dostal Z, Modriansky M. The effect of quercetin on microRNA expression: a critical review. Biomed Pap Med Fac Univ Palacky Olomouc Czech Repub. 2019:163(2):95-106.

20. Hosseinahli N, Aghapour M, Duijf PHG, Baradaran B. Treating cancer with microRNA replacement therapy: a literature review. J Cell Physiol. 2018; 233(8):5574-88

21. Zhang Y, Bai R, Liu C, et al. MicroRNA single-nucleotide polymorphisms and diabetes mellitus: a comprehensive review. Clin Genet. 2019:95(4):451-61.

22. Ou L, Sun $T$, Cheng $Y$, et al. MicroRNA-214 contributes to regulation of necroptosis via targeting ATF4 in diabetes-associated periodontitis. J Cell Biochem. 2019.

23. Perri $R$, Nares $S$, Zhang S, Barros SP, Offenbacher S. MicroRNA modulation in obesity and periodontitis. J Dent Res. 2012;91(1):33-8. 
24. Jiang $S$, Hu Y, Deng $S$, et al. miR-146a regulates inflammatory cytokine production in Porphyromonas gingivalis lipopolysaccharide-stimulated B cells by targeting IRAK1 but not TRAF6. Biochim Biophys Acta Mol Basis Dis. 2018;1864(3):925-33.

25. Li M, Zhou Y, Feng G, Su SB. The critical role of Toll-like receptor signaling pathways in the induction and progression of autoimmune diseases. Curr Mol Med. 2009;9(3):365-74

26. Kawai T, Akira S. Toll-like receptors and their crosstalk with other innate receptors in infection and immunity. Immunity. 2011;34(5):637-50.

27. Song GG, Kim JH, Lee YH. Toll-like receptor (TLR) and matrix metalloproteinase (MMP) polymorphisms and periodontitis susceptibility: a meta-analysis. Mol Biol Rep. 2013;40(8):5129-41.

28. Lin J, Bi L, Yu X, et al. Porphyromonas gingivalis exacerbates ligatureinduced, RANKL-dependent alveolar bone resorption via differential regulation of Toll-like receptor 2 (TLR2) and TLR4. Infect Immun. 2014;82(10): 4127-34.

29. Lin M, Hu Y, Wang Y, Kawai T, Wang Z, Han X. Different engagement of TLR2 and TLR4 in Porphyromonas gingivalis vs. ligature-induced periodontal bone loss. Braz Oral Res. 2017;31:e63.

30. Fuller K, Murphy C, Kirstein B, Fox SW, Chambers TJ. TNFalpha potently activates osteoclasts, through a direct action independent of and strongly synergistic with RANKL. Endocrinology. 2002;143(3):1108-18.

31. Jarry CR, Duarte PM, Freitas FF, et al. Secreted osteoclastogenic factor of activated T cells (SOFAT), a novel osteoclast activator, in chronic periodontitis. Hum Immunol. 2013;74(7):861-6.

32. Rifas L, Weitzmann MN. A novel T cell cytokine, secreted osteoclastogenic factor of activated $T$ cells, induces osteoclast formation in a RANKLindependent manner. Arthritis Rheum. 2009;60(11):3324-35.

33. Ukai T, Yumoto H, Gibson FC 3rd, Genco CA. Macrophage-elicited osteoclastogenesis in response to bacterial stimulation requires Toll-like receptor 2-dependent tumor necrosis factor-alpha production. Infect Immun. 2008;76(2):812-9.

34. Quinn SR, O'Neill LA. A trio of microRNAs that control Toll-like receptor signalling. Int Immunol. 2011;23(7):421-5.

35. Brudecki L, Ferguson DA, McCall CE, El Gazzar M. MicroRNA-146a and RBM4 form a negative feed-forward loop that disrupts cytokine mRNA translation following TLR4 responses in human THP-1 monocytes. Immunol Cell Biol. 2013;91(8):532-40

36. Hu Y, Yu P, Yu X, Hu X, Kawai T, Han X. IL-21/anti-Tim1/CD40 ligand promotes B10 activity in vitro and alleviates bone loss in experimental periodontitis in vivo. Biochim Biophys Acta. 2017.

37. Liu Z, Hu Y, Yu P, et al. Toll-like receptor agonists Porphyromonas gingivalis LPS and CPG differentially regulate IL-10 competency and frequencies of mouse B10 cells. J Appl Oral Sci. 2017:25(1):90-100.

38. Tu S, Zhong D, Xie W, Huang W, Jiang Y, Li Y. Role of toll-like receptor signaling in the pathogenesis of graft-versus-host diseases. Int J Mol Sci. 2016;17(8).

39. Gaddis DE, Michalek SM, Katz J. TLR4 signaling via MyD88 and TRIF differentially shape the CD4+ T cell response to Porphyromonas gingivalis hemagglutinin B. J Immunol. 2011:186(10):5772-83.

40. Zhang P, Liu J, Xu Q, et al. TLR2-dependent modulation of osteoclastogenesis by Porphyromonas gingivalis through differential induction of NFATc1 and NF-kappaB. J Biol Chem. 2011;286(27):24159-69.

41. Xie YF, Shu R, Jiang $S Y$, et al. miRNA-146 negatively regulates the production of pro-inflammatory cytokines via NF-kappaB signalling in human gingival fibroblasts. J Inflamm (Lond). 2014;11(1):38.

42. Zhang J, Jia G, Liu Q, et al. Silencing miR-146a influences B cells and ameliorates experimental autoimmune myasthenia gravis. Immunology. 2015;144(1):56-67.

43. Meisgen F, Xu Landen N, Wang A, et al. MiR-146a negatively regulates TLR2induced inflammatory responses in keratinocytes. J Invest Dermatol. 2014; 134(7):1931-40.

44. Zhang B, Wang A, Xia C, Lin Q, Chen C. A single nucleotide polymorphism in primary-microRNA-146a reduces the expression of mature microRNA146a in patients with Alzheimer's disease and is associated with the pathogenesis of Alzheimer's disease. Mol Med Rep. 2015;12(3):4037-42.

\section{Publisher's Note}

Springer Nature remains neutral with regard to jurisdictional claims in published maps and institutional affiliations.

\section{Submit your manuscript to a SpringerOpen ${ }^{\circ}$ journal and benefit from:}

- Convenient online submission

- Rigorous peer review

- Open access: articles freely available online

- High visibility within the field

- Retaining the copyright to your article

Submit your next manuscript at $\boldsymbol{\nabla}$ springeropen.com 